\title{
Carrier generation using a dual-frequency distributed feedback waveguide laser for phased array antenna (PAA)
}

\author{
M. R. H. Khan ${ }^{1,2^{*}}$, M. F. Islam¹, G. Sarowar ${ }^{1}$, T. Reza ${ }^{1}$ and M. A. Hoque ${ }^{1}$
}

\begin{abstract}
Background: Carrier generation based on optical heterodyning techniques where a beat signal is generated from the mixing of two light sources can be interesting solution due to ease on tunability. The free running heterodyning scheme benefits with a very wide tuning capability and the generated carrier can be freely adjusted. However, the drawbacks come in the form of frequency stability. Heterodyning of two optical frequencies coming from a single laser cavity has shown its potential on improvement of frequency stability.

Methods: The impact of the laser parameters (i.e., optical power and linewidth) on the quality of the generated carrier (i.e., phase/frequency stability and carrier-to-noise ratio) is analyzed for a $25 \times 64$ elements PAA system. An optically generated carrier signal using a dual-frequency distributed feedback waveguide laser in ytterbium doped aluminum oxide $\left(\mathrm{Al}_{2} \mathrm{O}_{3}: \mathrm{Yb}^{3+}\right)$ is analyzed and experimentally demonstrated in this paper. The carrier signal is used for downconversion of the signal received from a phased array antenna (PAA) of a DVB-S (Digital Video Broadcasting-Satellite) system.

Results: An optical frequency locked loop (OFLL) to stabilize the generated carrier is implemented which results in a microwave frequency at $\sim 14 \mathrm{GHz}$ with a phase noise of $-75 \mathrm{dBc} / \mathrm{Hz}$ at $1 \mathrm{MHz}$ offset from the center frequency and gives a loop settling time of $12 \mu$ s. By using the proposed OFLL, the long term and the short term frequency stability of the generated carrier has an Allan deviation of more than $1 \times 10^{-10}$ for an averaging time of $1000 \mathrm{~s}$ and a standard deviation of $39.4 \mathrm{kHz}$, respectively. The lasers linewidth should be in the order of tens of $\mathrm{Hz}$, with a maximum relative intensity noise (RIN) of $-107 \mathrm{~dB} / \mathrm{Hz}$ and a minimum optical power of $0.94 \mathrm{~mW}$.

Conclusions: The optical carrier generation by OFLL using a DFL to comply with the requirements of the standard carrier used in commercial LNBs has been investigated. The detailed analysis of the OFLL scheme is presented. Specifications requirement on carrier power, linewidth and relative intensity noise of the optically generated carrier have been addressed. The optical carrier generation is experimentally demonstrated and the requirements on CNR have been determined by measuring the noise floor and the optical carrier power. The measured values are compared with the calculated values and found to be very close.
\end{abstract}

Keywords: Phased array antenna, Optical heterodyning, Carrier generation, $K_{u}$-band, Laser linewidth, Stability, Optical frequency locked loop (OFLL), Dual frequency laser (DFL)

*Correspondence: rhkhan@iut-dhaka.edu

'Department of Electrical and Electronic Engineering, Islamic University of Technology, Gazipur 1704, Dhaka, Bangladesh

${ }^{2}$ Centre for Telematics and Information Technology (CTIT),

Telecommunication Engineering group, Faculty of EEMCS, University of

Twente, 7500 AE, Enschede, The Netherlands 


\section{Background}

The application of phase array antennas (PAA) to many satellite services, ranging from digital radio and TV broadcasts to broadband internet services, has been increasingly addressed in the last few years [1,2]. Potential applications especially regard mobile environments (cars, trains, boats, airplanes). A PAA is a set of multiple antennas, called antenna element (AE). Modern PAA consists of a large number of small antenna elements (AEs) and it can achieve the same gain of a large mechanically steerable antenna [3]. Recently, the use and advantages of PAA system for satellite communications systems have been thoroughly described in literature [4-6]. When installed on a vehicle a PAA system can be an advantageous solution for receiving satellite signals due to the flatness of its shape hence provides low aerodynamic profile [2] and to the fact that it can be electronically beamsteered, not requiring continuous mechanical realignment. Additional to that are the possibilities for beam-shaping, tracking and multi-beam reception [7].

The satellite signal received by the $\mathrm{AE}$ of the PAA is downconverted into lower frequency to ease on amplification and further processing. Downconversion is done by mixing the received signal with a carrier signal at an RF mixer placed at each $\mathrm{AE}$. To maintain phase synchronization among the carrier signals, the distribution of carrier signal from a central source is essential. The distribution of carrier signal by means of coaxial cable or waveguide is limited by the transmission line losses and their bulk size. Photonics offer an interesting alternative because these systems take advantage of the extremely low transmission and distribution losses $0.2 \mathrm{~dB} / \mathrm{km}$ in $1.5 \mu \mathrm{m}$ and $1 \mathrm{~dB} / \mathrm{km}$ in $1 \mu \mathrm{m}$ of the standard single-mode fiber (SMF). In addition to that, optical fiber cables are compact, lightweight and provide wide bandwidth and immunity to electromagnetic interference [8]. This approach has been proven as a successful solution in many deployed systems for instance radio-astronomy $[9,10]$ and satellites for remote sensing [11]. In order to use an optically generated carrier in a satellite reception system it must meet the criteria of that of the specifications given for commercially available electronic carrier. The crucial challenge for optically generated carrier is to meet the requirements in term of low phase noise, low drift, carrier-to-noise ratio (CNR) and wide tunability [12].

One of the methods to optically generate microwave carriers is to use optical heterodyning. In heterodyning between two separate single frequency lasers, all the phase noise from each laser is directly transferred into the microwave carrier [13]. Moreover, such scheme is rather bulky and suffers from large frequency drift of the microwave carriers. Heterodyning of two optical frequencies coming from a single laser cavity has shown its potential on improvement of frequency stability [14]. In this regard a dual-frequency laser (DFL) is interesting as the relative fluctuations between the two frequencies are low and can be used to generate a low-phase-noise microwave carrier due to the common-mode noise rejection effect $[13,15-17]$.

In this paper we consider an optically generated carrier based on heterodyning of a dual-frequency laser reported recently [14]. This laser is in ytterbium-doped aluminum oxide $\left(\mathrm{Al}_{2} \mathrm{O}_{3}: \mathrm{Yb}^{3+}\right)$ fabricated on a silicon substrate, which shows great potential for the monolithic integration of optically generated microwave sources. Here we present an efficient technique to lock a dual-frequency $\left(\mathrm{Al}_{2} \mathrm{O}_{3}: \mathrm{Yb}^{3+}\right)$ DFB channel waveguide laser using an OFLL in order to improve the free-running frequency and power stability of the optically generated carrier, while also reducing the phase noise. This approach is promising in terms of compactness, low phase noise and high stability. This paper demonstrates the feasibility of such a system to provide carrier signals.

The remainder of the paper is organized as follows. In "Methods" section, the proposed optically generated carrier for PAA system using a DFL is described and analyzed for the carrier generation criteria. "Results and discussion" section presents performance of the generated carrier. In the last section, the conclusions of this analysis are stated.

\section{Methods}

\section{System description}

The block diagram of the proposed antenna system using an optically generated carrier is presented in Fig. 1.

Since the received signal power density from the satellite is relatively low, the antenna should have a high gain and a low noise temperature to achieve the required CNR for $\mathrm{K}_{u}$-band satellite signals. This dictates a number of antenna elements of at least 1600 [1]. Those can be placed as 25 tiles of 64 AEs. More details of this PAA system have been described in [1]. In this paper we restrict ourself to the downconversion system of the DVB-S reception. The proposed downconversion system consists of a PAA, an optical distribution network (ODN) and the optical heterodyning scheme.

As a basic building block, we consider an $8 \times 8$ antenna tile consisting of 64 antenna elements (AEs), shown in Fig. 1a. The DVB-S signal received by each AE will be amplified by a low noise amplifier (LNA) and downconverted at the mixer using a centrally generated carrier signal.

The carrier is generated optically and also distributed by an optical distribution network (ODN) in order to take advantage of low loss distribution by means of standard single-mode fiber. Shown in Fig. 1b, the ODN is implemented in conjunction with an individual photodetector (PD) at each antenna tile. The carrier signal at the output of each PD is then distributed to each mixer. 


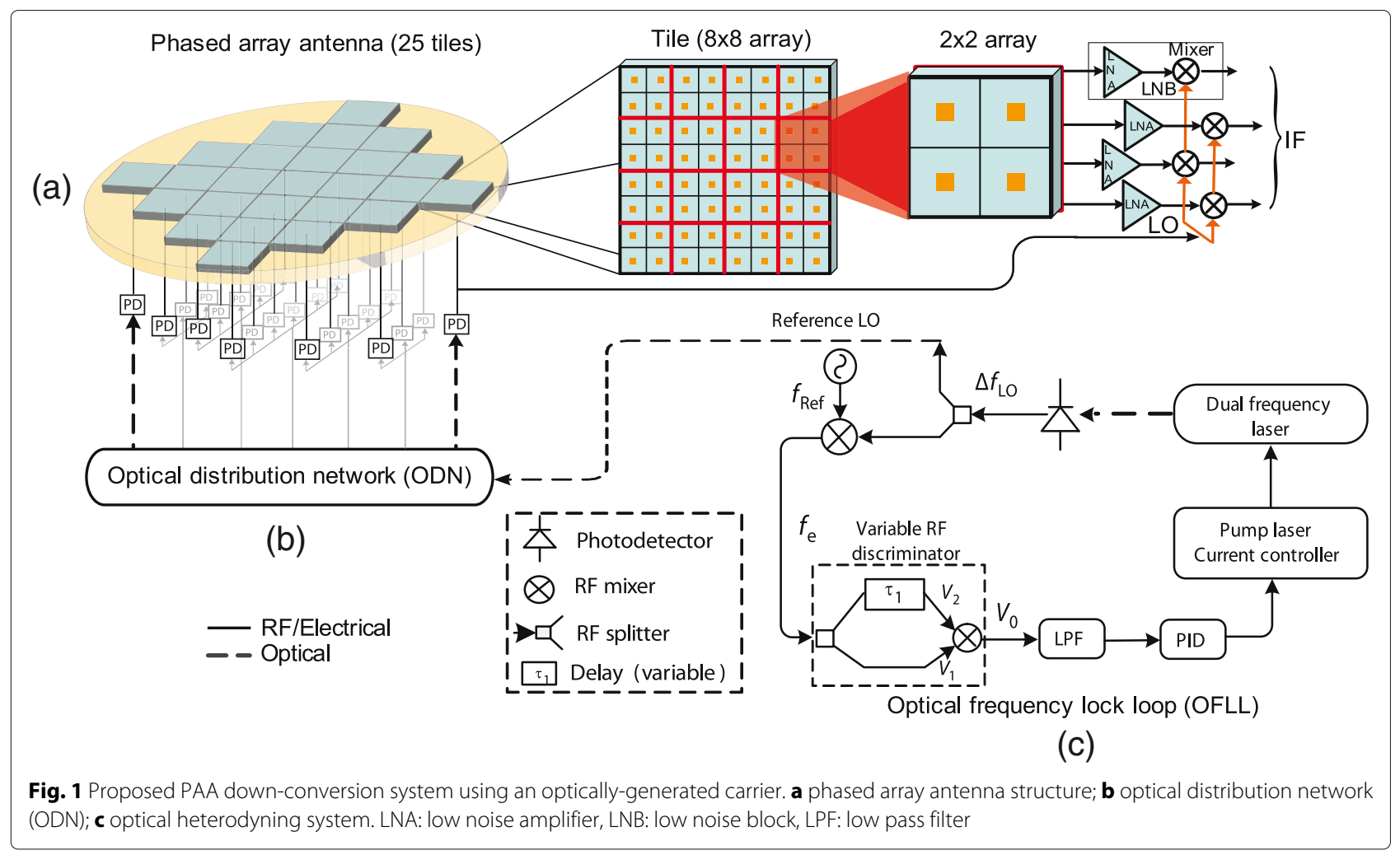

The optical heterodyning system that generates the carrier signal is shown in Fig. 1c. The DFL laser produces a beat frequency of $\approx 14 \mathrm{GHz}$ at the output of the PDs. This enables downconvertion of $10.7 \mathrm{GHz}$ to $12.75 \mathrm{GHz}$ DVB-S signal to a required intermediate frequency (IF) of $950 \mathrm{MHz}-2150 \mathrm{MHz}$. The frequency stabilization of the optically generated carrier is accomplished by an optical frequency lock loop (OFLL) explained in detail in "Stabilization of optically generated carrier signals" section.

\section{Dual frequency laser characteristic}

Operation of the optically pumped DFL is based on two localized quarter wavelength phase shifts in a straight channel waveguide DFB cavity. The schematic of the dual frequency DFB cavity, along with the longitudinal field distribution of the two respective laser wavelengths was calculated by using numerical integration methods explained in detail in [18] and is shown in Fig. 2. All calculations performed in this work were implemented in Matlab.

When two quarter-wavelength phase shifts are induced in a uniform waveguide Bragg grating, two resonances appear in the transmission stop-band of the device. These two resonances share a common cavity, which consists of both phase shifts, and the frequency spacing between the two resonances (lasing modes) depends on the spatial separation and values of the respective phase shifts in the cavity. The frequency spacing between the two laser modes is what ultimately determines the beat frequency at the output of the PD. For two discrete quarter-wavelength phase shifts, the frequency spacing between two resonances is given by [19]

$$
\Delta f=\frac{c}{8 n L}
$$

where $c$ is the speed of light, $L$ is the distance between the two phase shifts and $n$ is the effective refractive index of the guided mode. This implies that the beat frequency can be scaled to higher frequencies by reducing $L$. However, the stop band of the Bragg grating in the device has a width of approximately $30 \mathrm{GHz}$, which poses an upper-limit to the frequency separation of the two optical carriers, and thus the beat frequency, which can be generated with the current grating design.

When both phase shifts are varied symmetrically from a quarter-wavelength phase shift $\pi / 2$, such that one has a value of $\pi / 2-\Delta \theta$ and the other $\pi / 2+\Delta \theta$, then the two resonant wavelengths separate symmetrically from each other with respect to the Bragg wavelength, while they remain equivalent in terms of their amplitude and line width. In other words, the value of $\Delta \theta$ can be used to increase the frequency spacing between the two resonances as compared to the frequency spacing given by Eq. (1). 


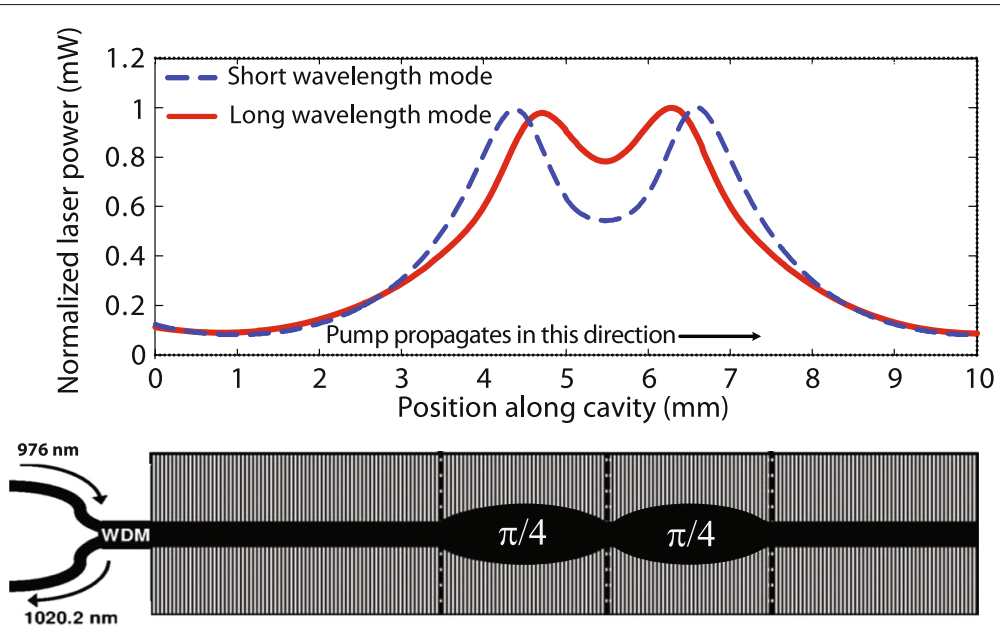

Fig. 2 Schematic of the dual frequency DFB cavity, along with the calculated longitudinal field distribution of the two respective laser wavelengths

Making use of standard lithography and a chlorine based reactive ion etching process, $2.5 \mu \mathrm{m}$ wide waveguides were fabricated in an $\mathrm{Al}_{2} \mathrm{O}_{3}: \mathrm{Yb}^{3+}$ waveguide layer with an ytterbium concentration of $5.8 \times 10^{2} \mathrm{~cm}^{-3}$, after which the waveguide layer was overgrown with a plasma enhanced chemical vapour deposited $\mathrm{SiO}_{2}$ cladding layer. A $10 \mathrm{~mm}$ long surface corrugated Bragg grating was defined by means of laser interference lithography and etched into the top surface of the $\mathrm{SiO}_{2}$ layer with reactive ion etching. The detail process of fabricating the waveguide is explained in [14]. In order to realize the two required quarter-wavelength phase shifts, two sections with $2 \mathrm{~mm}$ long adiabatic sinusoidal widening of the waveguide width are fabricated [20]. The two quarterwavelength phase shifts are centered at $4.5 \mathrm{~mm}$ and 6.5 $\mathrm{mm}$ respectively (as measured from the pumped end facet of the $1 \mathrm{~cm}$ long cavity). Based on coupled-mode theory, where the grating reflectivity at the Bragg wavelength is given by $R=\tanh ^{2}(\kappa L)$, where $\kappa L$ is the dimensionless quantity express the grating strength [21]. Each of the bragg reflectors was designed to have a grating coupling coefficient of $k=6 \mathrm{~cm}^{-1}$. Fabrication of the waveguide and Bragg gratings is described in detail in [14].

The experimental setup in Fig. 3 is used to characterize the DFL cavity. A 980/1030 nm wavelength division multiplexing (WDM) fiber was butt-coupled to the optical chip and fixed into position by the use of UV curable glue. The WDM fiber was deliberately misaligned with respect to the waveguide to reduce the effect of optical back reflections in the laser cavity. The $976 \mathrm{~nm}$ diode pump light was launched into the waveguides via the 980 $\mathrm{nm}$ port of the WDM fiber, while the laser emission was collected through the $1030 \mathrm{~nm}$ port, which also contained an isolator to further prevent optical back-reflections into the laser cavity (shown in Fig. 3). The back-reflections mainly originated from Fresnel reflections on the 1030 $\mathrm{nm}$ fiber facet and caused instabilities despite the use of a $22 \mathrm{~dB}$ optical isolator and an 8 degree angled fiber facet. The laser emission was sent via the WDM fiber to either a power meter, or a photodetector. The laser emission spectrum is centered at a wavelength of 1020 $\mathrm{nm}$ and a measurement with the $40-\mathrm{GHz}$ bandwidth PD (New Focus 1011) confirmed the dual-frequency operation with a beat signal at $\approx 14 \mathrm{GHz}$ shown in Fig. 4a. The operating wavelength of the DFL could be shifted to $1550 \mathrm{~nm}$ or $1330 \mathrm{~nm}$ by using different doping material as demonstrated in [20].

Since the fiber was misaligned with respect to the waveguide, it was not possible to measure exactly how much pump power was launched into the waveguide. Laser oscillation started at an estimated launched pump power of $2.5 \mathrm{~mW}$, after which the laser output power increased linearly to produce a pump-power limited output power of $0.28 \mathrm{~mW}$. Fig. 5a shows the measured laser power as a function of the diode pump current. Due to the misalignment of WDM fiber with respect to the waveguide the measured laser power is much lower than what is actually emitted out of the waveguide [22]. The beat frequency as a function of diode pump current is shown in Fig. $5 \mathrm{~b}$, where a change in pump current was measured to induce a frequency shift of $3.6 \mathrm{MHz} / \mathrm{mW}$ in the generated microwave beat signal.

The two side bands on either side of the main microwave peak are produced by relaxation oscillations from the two respective longitudinal modes (Fig. 4a). The relaxation oscillation frequencies were measured as a function of pump power and by comparing the onset of the respective relaxation oscillations it became apparent that one of the longitudinal modes had a $20 \%$ higher pump threshold than the other (Fig. 6). The shorter wavelength most 


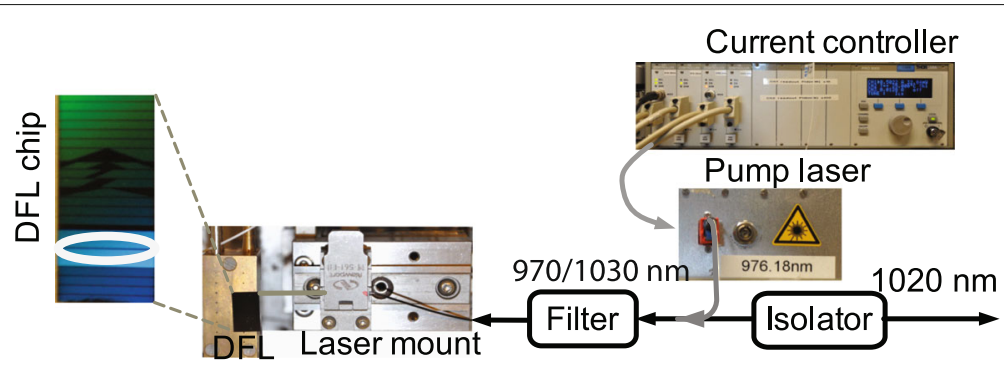

Fig. 3 DFL characterization setup

likely reaches threshold first, since it oscillates around the phase shift nearer to the pumped side of the chip where it receives more pump power than the longer wavelength [23].

The two relaxation-oscillation frequencies were used to calculate the Lamb's coupling constant given by [24]

$$
C_{\mathrm{lamb}}=\left[\frac{1-\left(f_{\mathrm{L}} / f_{\mathrm{R}}\right)^{2}}{1+\left(f_{\mathrm{L}} / f_{\mathrm{R}}\right)^{2}}\right]
$$

where $f_{\mathrm{L}}$ and $f_{\mathrm{R}}$ are the relaxation oscillation frequencies of the two respective longitudinal modes with $f_{\mathrm{L}}<f_{\mathrm{R}}$. The value of $C_{\text {lamb }}$ indicates whether there exists weak $(C \approx 0)$ or strong $(C \approx 1)$ mode competition between the two oscillating wavelengths. By using the measured relaxation oscillation frequencies as shown in Fig. 6, the value of $\mathrm{C}$ could be determined. Just above threshold of the second mode, $(C>0.5)$, indicating a relatively strong interaction and mode competition between the two longitudinal laser modes close to threshold. However, with increasing pump power $C_{\mathrm{lamb}}$ converges to a value of $C_{\mathrm{lamb}}=0.23$, indicating that the mode competition is rather weak, since each longitudinal mode was being amplified mostly by separate sections of the active medium. The weak longitudinal mode interaction was confirmed by the longitudinal field overlap between the two modes (Fig. 2), which was calculated to be 0.34 [14]. Moreover, for a period of 45 min the power of the microwave signal was stable within $\pm 0.35 \mathrm{~dB}[14]$.

The optical spectrum of the DFL is measured by an optical spectrum analyzer (OSA) (ANDO AQ6317) is shown in Fig. 4b. This optical spectrum was measured at a pump power of $35 \mathrm{~mW}$.

\section{Optical carrier generation}

Let us consider two optical modes $L_{1}$ and $L_{2}$ at frequencies $f_{\mathrm{L} 1}$ and $f_{\mathrm{L} 2}$, respectively. These two modes with the same polarization is combined. A beat signal is created at the output of the photodetector. In the absence of frequency noise, the photodetector output current, $I(t)$, is a sinusoidal signal with a frequency equal to the frequency difference between the two lasers, $f_{\mathrm{LO}}=f_{\mathrm{L} 1}-f_{\mathrm{L} 2}$. The output current from the detector can be expressed as [25]

$$
I(t)=r_{\mathrm{pd}}\left(P_{\mathrm{L} 1}+P_{\mathrm{L} 2}\right)+2 r_{\mathrm{pd}} \sqrt{P_{\mathrm{L} 1} P_{\mathrm{L} 2}} \cos \left(\left[2 \pi f_{\mathrm{LO}} t+\phi_{\mathrm{LO}}\right]\right)
$$

where $r_{\mathrm{pd}}$ is the responsivity of the photodetector, $P_{\mathrm{L} 1}$ and $P_{\mathrm{L} 2}$ are the optical power of the two modes. The phase variation $\phi_{\mathrm{LO}}=\phi_{1}(t)-\phi_{2}(t)$ is the difference of the

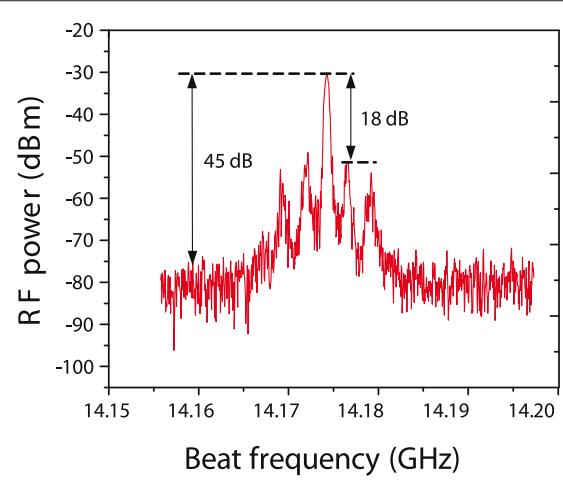

(a)

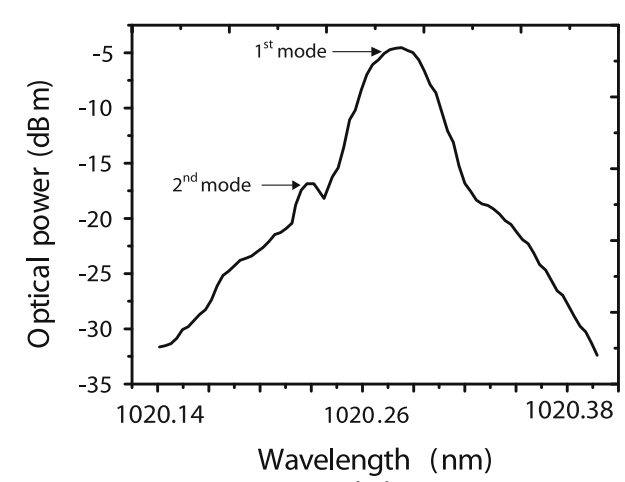

(b)

Fig. 4 DFL spectrum of (a) optical (b) RF carrier 


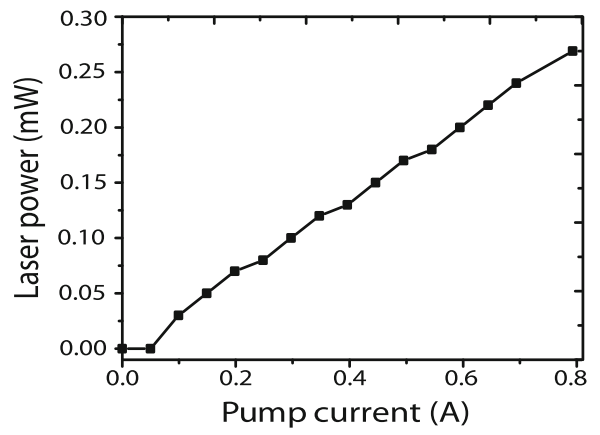

(a)

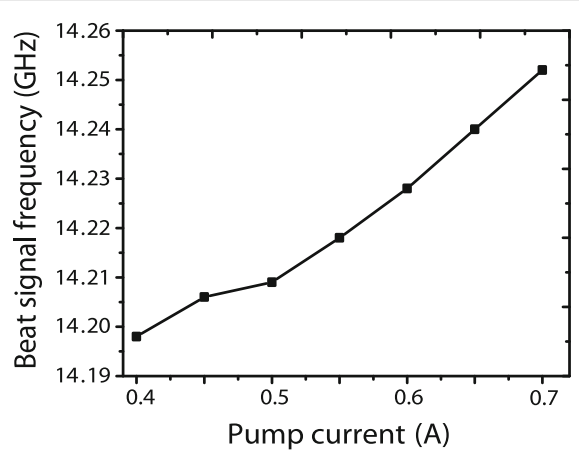

(b)

Fig. 5 Dual-frequency laser characteristic (a) laser power as a function of pump current (b) laser frequency as a function of pump current

independent phase variations of the individual lasers and $2 r_{\mathrm{pd}} \sqrt{P_{\mathrm{L} 1} P_{\mathrm{L} 2}}$ is the amplitude of the generated carrier. The power spectral density (PSD) of the photodetector output current, $I(t)$ is expressed as $[25,26]$

$$
S_{I}(f)=2 r_{\mathrm{pd}}^{2} P_{\mathrm{L} 1} P_{\mathrm{L} 2} \frac{\frac{1}{\pi \Delta v_{\mathrm{LO}}}}{1+\left[\frac{f-f_{\mathrm{LO}}}{\Delta v_{\mathrm{LO}}}\right]^{2}}
$$

where the beat linewidth of the optically generated carrier, $\Delta v_{\mathrm{LO}}=\Delta v_{1}+\Delta v_{2}$ is equal to the sum of the two linewidths of the lasers $[27,28]$. The PSD of the optically generated carrier is a Lorentzian centered at $f_{\mathrm{LO}}$. Due to the laser phase noise, the spectral linewidth of the laser broadens into a Lorentzian shape and ultimately broadens the linewidth of the optically generated carrier.

The RF power of the carrier signal delivered to a load can be calculated using the following equation:

$$
P_{\mathrm{LO}}=\left\langle I^{2}(t)\right\rangle R_{\mathrm{L}}=\frac{1}{2}\left(2 r_{\mathrm{pd}} P_{\mathrm{L}}\right)^{2} R_{\mathrm{L}}=\frac{1}{2} I_{\mathrm{av}}^{2} R_{\mathrm{L}}
$$

where the symbol $\langle\cdot\rangle$ denotes average, $I$ and $R_{\mathrm{L}}$ are the current and load impedance at the output of the photode-

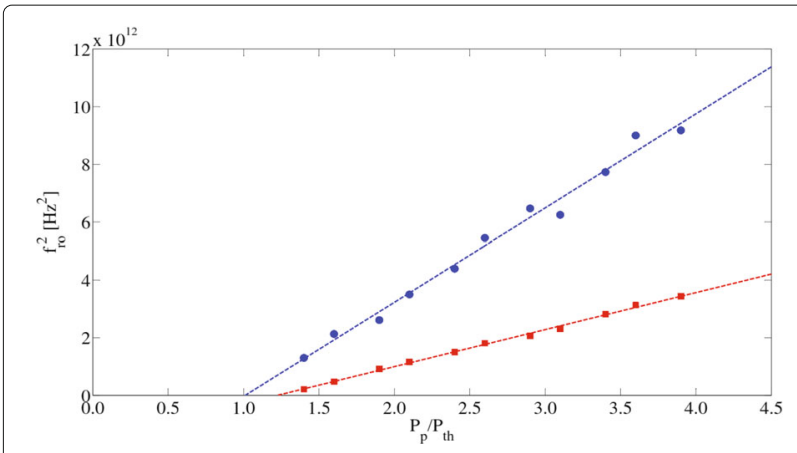

Fig. 6 Square of the relaxation oscillation frequency $\left(f_{\text {ro }}\right)$ of the two longitudinal laser modes as a function of $P_{p}=P_{\text {th }}$, with $P_{p}$ the pump power and $P_{\text {th }}$ the threshold pump power of the lowest threshold mode tector, respectively, and $I_{\mathrm{av}}$ is the average photo-current expressed as:

$$
I_{\mathrm{av}}=r_{\mathrm{pd}} P_{\mathrm{T}}=r_{\mathrm{pd}}\left(P_{\mathrm{L} 1}+P_{\mathrm{L} 2}\right)=2 r_{\mathrm{pd}} P_{\mathrm{L}}
$$

\section{Criteria for the optically generated carrier}

In order to use the optically generated carrier in a DVB-S system its performance must meet the criteria for power, phase noise and frequency stability. The parameter values that are useful for our analysis are taken from the DVB-S standard [29], specifically the phase noise values shown in Table 1.

\section{Linewidth criteria of optically generated carrier}

Close to the carrier frequency, the performance of the generated carrier is determined by the linewidth of the optical carrier. The ratio between Eqs. (4) and (5) gives the single sideband (SSB) phase noise expressed in $\mathrm{dBc} / \mathrm{Hz}$ [30]. Calculating the SSB phase noise curve for various offset frequencies and comparing it to the values from Table 1, one can determine the linewidth of the optical carrier to match the DVB-S requirements. It is evident that the parameters in Table 1 shows a Lorentzian decay of the SSB phase noise.

\section{Carrier-to-noise ratio criteria of photonic carrier}

Beyond $1 \mathrm{MHz}$ offset frequency from the carrier, it is the noise floor that determines the system performance. To

Table 1 Carrier specifications for DVB-S receiver

\begin{tabular}{ll}
\hline Carrier Parameters & Values \\
\hline Frequency range & $9.75 \mathrm{GHz}$ or $10.6 \mathrm{GHz}$ \\
Single sideband & $-50 \mathrm{dBc} / \mathrm{Hz} @ 1 \mathrm{kHz}$ \\
(SSB) phase noise & $-70 \mathrm{dBc} / \mathrm{Hz} @ 10 \mathrm{kHz}$ \\
& $-90 \mathrm{dBc} / \mathrm{Hz} @ 100 \mathrm{kHz}$ \\
& $-110 \mathrm{dBc} / \mathrm{Hz} @ 1 \mathrm{MHz}$ \\
& $5 \mathrm{MHz}$
\end{tabular}


analyze the impact of noise in this heterodyning system, it is useful to consider all noise sources in the photonic system. The system is affected by noise, comprising thermal noise, shot noise and relative intensity noise (RIN).

According to the carrier specifications in Table 1, at $1 \mathrm{MHz}$ offset frequency the generated carrier signal has to have less than $-110 \mathrm{dBc} / \mathrm{Hz}$ phase noise. To keep this value for the optically generated carrier the noise floor must not exceed this limit. To maintain a noise floor of $110 \mathrm{~dB}$ below the carrier power, i.e., CNR, a minimum carrier power is required. Equation 5 shows that the minimum carrier power ultimately sets the minimum laser power in the heterodyning operation.

\section{Stabilization of optically generated carrier signals}

Several investigations and experiments show that the beat signal generated by a free-running heterodyning system suffers substantial frequency drift [16]. The frequency of a laser generally drifts hundreds of $\mathrm{MHz}$ in an ordinary environment [31]. According to the DVB-S specification [29], the maximum allowable frequency drift of the generated carrier is $5 \mathrm{MHz}$ [32]. Therefore, the optically generated carrier needs to be frequency stabilized.

\section{Optical frequency locked loop schemes}

For the purpose of stabilizing the optically generated carrier, the beat signal is often mixed down to a lower frequency which can be easily processed. One of the main differences among the various optical frequency locking schemes which have been developed in the last decades, is the method used to convert the beat frequency or the downconverted beat frequency to proportional error metric. This error metric controls the frequency of the socalled slave laser maintaining a highly precise frequency offset from a reference laser, labeled as master laser. Previously, a frequency locking approach converted the beat frequency to a proportional voltage by an electronic circuit [33]. The voltage is then compared to a reference voltage in an electronic comparator, which sets the beat frequency. Another frequency locking scheme uses a frequency divider (prescaler) on the beat frequency to down scale it before processing the error signal [34]. Both of the schemes use active electronic components as frequency to voltage converter (FVC) so it degrades the system dynamics (i.e., loop bandwidth, loop response time). A simple OFLL technique based on the concept presented by Schunemann et al. [35] where an RF discriminator is used as a FVC is proposed. In this discriminator a variable delay line is used as a frequency dependent phase shifter in conjunction with a phase detector to facilitate beat frequency tuning. Our proposed scheme uses all passive RF components (RF power splitter, variable delay line, $\mathrm{RF}$ mixer) in the FVC to improve the dynamics of the loop.
The block diagram of the proposed OFLL is presented in Fig. 7.

\section{Proposed optical frequency locked loop scheme}

The beat signal provided by a $40 \mathrm{GHz}$ bandwidth photodetector (New Focus 1011) is amplified using a commercial RF amplifier. A power splitter (MiniCircuits ZFRSC-123$S$ ) is used to tap the beat signal for monitoring. The output signal from one of the outputs of the power splitter is mixed with a reference signal, $f_{\text {Ref, }}$ at frequency around $14 \mathrm{GHz}$ provided by a signal generator (Agilent PSG E8267D). A downconverted signal, $V_{\mathrm{IF}} \cos \left(2 \pi f_{\mathrm{IF}} t\right)$, is produced at the output of the RF mixer (MiniCircuits ZX05-153LH-S) where $V_{\mathrm{IF}}$ is the amplitude of the downconverted signal and $f_{\mathrm{IF}}$ is the intermediate frequency (IF) given by $f_{\mathrm{IF}}=\Delta f_{\mathrm{LO}}-f_{\text {Ref. }}$. The signal is then passed through a variable frequency discriminator. In the frequency discriminator the signal is split into two equal parts by an RF splitter and recombined at a mixer (MiniCircuits SBL 48), after one part has been delayed by a variable delay line (Narda model 3752). Hence,

$$
V_{1}=\frac{V_{\mathrm{IF}}}{2}\left[\cos \left(2 \pi f_{\mathrm{IF}} t\right)\right] ; V_{2}=\frac{V_{\mathrm{IF}}}{2}\left[\cos \left(2 \pi f_{\mathrm{IF}}\left(t-\tau_{1}\right)\right)\right]
$$

where $V_{1}$ and $V_{2}$ are the signals combined in the mixer and $\tau_{1}$ is the time delay. The resultant signal of the frequency discriminator at the output of the double balanced mixer (MiniCircuits ZFM-2), $V_{0}$, is [36]

$$
V_{0}=\frac{V_{\mathrm{IF}}}{\pi}\left[\cos \left(2 \pi f_{\mathrm{IF}} \tau_{1}\right)+\cos \left(2 \pi f_{\mathrm{IF}}\left(2 t-\tau_{1}\right)\right)+\ldots\right]
$$

The high-frequency terms $\left(2^{\text {nd }}\right.$ and successive terms between the brackets) are eliminated by the cut-off frequency of the current controller, $f_{\mathrm{c}}$, of the pump laser given as $100 \mathrm{kHz}^{1}$ which is lower than that of the $10 \mathrm{MHz}$ of the LPF. In this case the current controller of the pump laser will ultimately dictate the frequency response and bandwidth of the feedback loop.

\section{Characterization of the components}

From the schematic of the proposed OFLL (in Fig. 7), one can notice that the dynamics of the loop will be influenced by the characteristics, namely the frequency response and the conversion factor, of the various components used in the loop. In this section the characteristics of the FVC is investigated.

The discriminator signal at the output of the LPF as a function of the beat frequency for a delay of $\tau_{1}=3 \mathrm{~ns}$ is shown in Fig. 8. The delay of 3 ns was realized by using a passive RF component, namely a phase shifter (Narda model 3752). As shown in this figure, the OFLL allows stable locking to several values of the nulls which are 


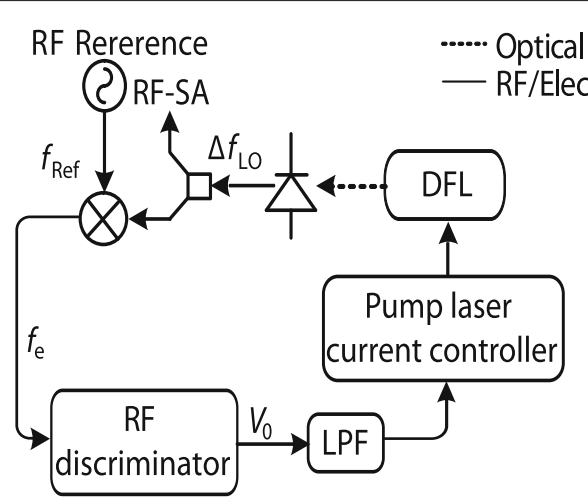

(a)

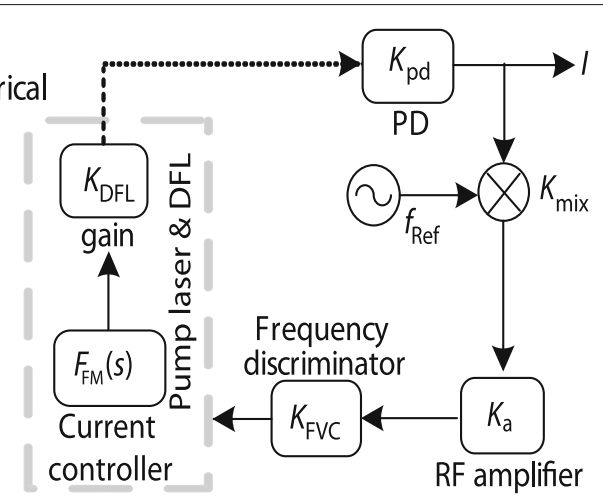

(b)

Fig. 7 OFLL to stabilize dual-frequency laser. a schematic diagram (b) linearized s-domain representation

spaced by $1 / \tau_{1}$ corresponding to the zero crossings of the error signal. The difference in frequency (offset) between the first null (the point where the beat frequency being locked) from the reference frequency is given by

$$
\Delta f=\frac{1}{2 \tau_{1}}
$$

The purpose of the RF discriminator used in the OFLL is to convert the frequency to its input to a proportional voltage. The frequency-to-voltage conversion factor, $K_{\mathrm{FVC}}$, for the frequency discriminator is maximum at the closest nulls to the reference frequency (point A and B in Fig. 8). From Eq. (9), a higher delay will reduce the frequency offset of the null from the reference frequency and will increase the slope at the nulls which ultimately will give a higher conversion of $K_{\mathrm{FVC}}$. The error signal at the output of the low pass filter (LPF) is sent to the pump laser (976.18 nm) in order to control its current and, consequently, the optical pump power. A change in the pump power changes the thermally induced chirp in the Bragg grating, which, in turn, changes the frequency separation between the two laser modes. The commercial pump laser current controller (Thorlabs PRO8000) of the DFL has an external tuning port having a voltage-to-current

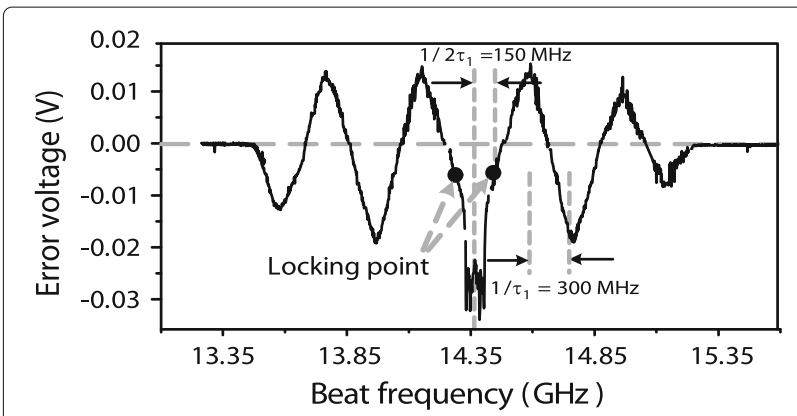

Fig. 8 Error signal as function of beat frequency conversion factor of $200 \mathrm{~mA} / \mathrm{V}$. For the pump powers at which the DFL operates, the optically generated microwave carrier increases linearly with pump current at a rate of $180 \mathrm{kHz} / \mathrm{mA}$. This gives a voltage-to-frequency conversion, $K_{\mathrm{DFL}}$, of $36 \mathrm{kHz} / \mathrm{mV}$ in Fig. 7 .

The various parameters and their values used in our analysis are summarized in Table 2.

\section{Optical frequency lock loop analysis}

The proposed OFLL in Fig. 7a can be represented in a generic model for a feedback system and presented as Laplace domain (s-domain) representation [37] of OFLL in Fig. 7b.

In Fig. 7, the optical and the electrical connections are represented by dotted and solid lines, respectively. The optical field from the DFL is represented by $E_{\mathrm{L}}$. The gain of the PD can be defined as $K_{\mathrm{pd}}=r_{\mathrm{pd}}$ where $r_{\mathrm{pd}}$ is the responsivity of the PD. The output beat frequency from the PD is mixed with a reference frequency, $f_{\text {Ref. The RF }}$ mixer has a conversion loss of $K_{\mathrm{mix}}$. The downconverted signal is then amplified by an RF amplifier with a gain of $K_{\mathrm{a}}$. An RF frequency discriminator converts the frequency to its proportional voltage with a conversion factor

Table 2 Various loop parameters

\begin{tabular}{|c|c|c|c|}
\hline Parameter & Value & Dimension & Notation \\
\hline Responsivity of the PD & 0.4 & $\mathrm{~A} / \mathrm{W}$ & $r_{\mathrm{pd}}$ \\
\hline Conversion loss of the mixer & -5.5 & $d B$ & $K_{\text {mix }}$ \\
\hline Total gain of the RF amplifiers & variable & $d B$ & $K_{\mathrm{a}}$ \\
\hline $\begin{array}{l}\text { Frequency-to-voltage conversion } \\
\text { factor of the RF discriminator }\end{array}$ & 0.15 & $\mathrm{mV} / \mathrm{MHz}$ & $K_{\mathrm{FVC}}$ \\
\hline $\begin{array}{l}\text { Cut-off frequency of the current } \\
\text { controller of the pump laser }\end{array}$ & 100 & $\mathrm{kHz}$ & $f_{c}$ \\
\hline $\begin{array}{l}\text { Voltage-to-frequency conversion } \\
\text { factor of the current controller }\end{array}$ & 36 & $\mathrm{MHz} / \mathrm{mV}$ & $K_{\mathrm{DFL}}$ \\
\hline
\end{tabular}


of $K_{\mathrm{FVC}}$. The error voltage is passed through a LPF to filter out the high frequency components and is then applied to the current controller of the pump laser. The pump laser acts as a current-controlled oscillator and the change in output optical power is a function of the frequency components of the input current modulation. The frequency response of the current controller is indicated by $F_{\mathrm{FM}}(f)$ and its Laplace transform is denoted by $F_{\mathrm{FM}}(s)$. The external voltage-to-optical power conversion factor is denoted by $K_{\mathrm{SL}}$. As the bandwidth of the current controller will dominate in the loop so its frequency response will be considered in the s-domain model.

From the linearized s-domain representation of the OFLL the closed-loop transfer function of the OFLL is [37]

$$
H(s)=\frac{4 \pi^{2} f_{\mathrm{n}}^{2}}{s^{2}+s \zeta 2 \pi f_{\mathrm{n}}+4 \pi^{2} f_{\mathrm{n}}^{2}\left(1+\frac{1}{K}\right)}
$$

where $K=K_{\mathrm{pd}} K_{\mathrm{mix}} K_{\mathrm{a}} K_{\mathrm{FVC}} K_{\mathrm{DFL}}$ is the total gain and $\zeta$ and $f_{\mathrm{n}}$ represent the damping factor and the natural frequency of the loop, respectively, is expressed by following Eqs. [37]:

$$
\begin{gathered}
\zeta=\frac{f_{\mathrm{c}}}{f_{\mathrm{n}}} \\
f_{\mathrm{n}}=f_{\mathrm{r}} \sqrt{K}
\end{gathered}
$$

\section{Results and discussion}

In this section we will present the experimental results on various performance criteria of an optically generated carrier, namely the long term frequency stability, the short term frequency stability, loop response and the phase noise.

\section{Long term frequency stability}

A free-running microwave frequency, $f_{\mathrm{LO}}$, deviates from the required frequency, $f_{\text {ideal }}$, with variable direction and speed. In order to express the long term frequency stability of a carrier, Allan deviation, $\sigma_{y}(\tau)$, is often used. Allan deviation was designed to extrapolate the drift of a system at infinity from finite measurements. Allan deviation has proven itself a valuable tool to quantify the stability of optically generated microwave carrier [38]. The Allan deviation of the relative frequency error $y(t)=1-$ $\left(\Delta f_{\mathrm{LO}} / f_{\text {ideal }}\right)$ is defined by.

$$
\sigma_{y}(\tau)=\left[\frac{1}{2(M-1)} \sum_{i=1}^{M-1}\left(\bar{y}_{i+1}-\bar{y}_{i}\right)^{2}\right]^{1 / 2}
$$

where $y_{i}$ is the $i^{\text {th }}$ of $M$ fractional frequency values averaged over the measurement (sampling) interval, $\tau$.

The measured frequency stability of the generated carrier is expressed in Allan deviation in Fig. 9 for both locked and free-running condition.

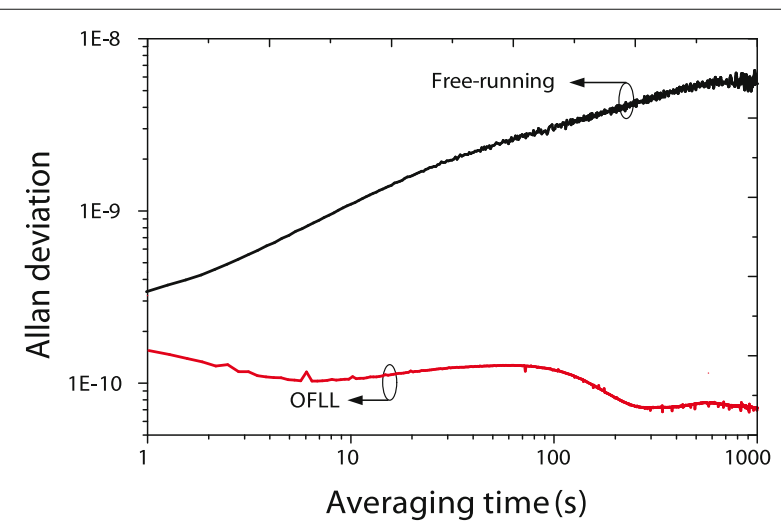

Fig. 9 Allan deviation of the frequency stability of the DFL under free-running and locked condition

The Allan deviation for the free-running condition increases from $3.5 \times 10^{-10}$ at an averaging time of $\tau=1 \mathrm{~s}$ to $5 \times 10^{-9}$ at an averaging time of $\tau=1000 \mathrm{~s}$. However, the long-term frequency stability of the DFL could be improved to a value of $1 \times 10^{-10}$ for an averaging time, $\tau$, of $1000 \mathrm{~s}$ by implementing the OFLL. These results emphasize the effectiveness of using the OFLL for improving the long-term stability of the free-running microwave carrier by a 50 fold improvement in the frequency stability at $1000 \mathrm{~s}$ averaging time. The long-term frequency stability of the OFLL shows batter performance than a typical quartz oscillator (Wenzel 501-04623E) having a frequency stability of Allan deviation of $4 \times 10^{-7}$ [39]. Moreover, this performance is also comparable to recently reported radio frequency (RF) clock signals transmission over $100 \mathrm{~m}$ via fiber link results in an Allan deviation of $1.31 \times 10^{-10}$ for an averaging time of $1 \mathrm{~s}[40]$.

\section{Short term frequency stability}

In addition to the requirement of long term frequency stability for the application like RF clock/reference signal transmission, radio astronomy or microwave carrier generation and distribution of a PAA, these applications also require very good short-term frequency stability [41]. The improvement of the short-term frequency stability also improves the phase noise performance of the generated carrier [42]

In order to investigate and analyze the short-term frequency stability, it is important to observe the frequency stability with very short sampling duration ( $\mu$ s or lower). The maximum observation speed of our setup is limited by data recording speed of the simulator and it is $100 \mathrm{~ms}$. This speed is not enough for short term frequency analysis, as an alternative we have produced a spectrogram of the generated microwave in a very short time period (i.e., in $\mu \mathrm{s})$. The schematic to produce such a spectrogram is shown in Fig. 10. 


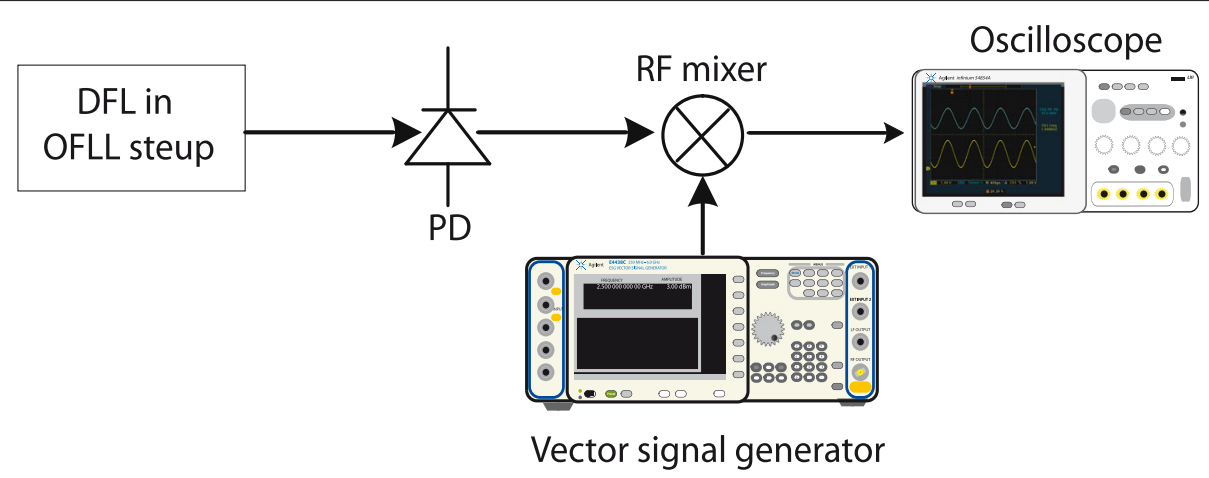

Fig. 10 Schematic of the setup used to produce the spectrogram. DFL: dual frequency laser, OFLL: optical frequency lock loop, PD: photodetector

The procedure of producing such a spectrogram is as follows. The microwave carrier at the output of the photodetector was mixed with a high-purity, stable RF reference signal produced by a vector signal generator (Agilent PSG E8267D) with a sub-Hz line width, which was produced by a microwave carrier generator. This reference signal was set within a few $\mathrm{MHz}$ offset of the $14.21 \mathrm{GHz}$ microwave beat signal produced by the DFL. Since the reference signal was much narrower than the laser beat signal, mixing of the two signals produced a convoluted signal which was nearly identical in shape to the original microwave beat signal produced by the DFL, but shifted to the $\mathrm{MHz}$ range, so that a time trace of this down converted signal could be measured with an oscilloscope (Agilent infinium 54854A). A Discrete Fourier transformation (DFT) over duration of a short time frame $(\mu \mathrm{s})$ was then performed on that time trace of the down-converted signal [13], This will give the frequency spectrum of the time domain signal, with various center frequency due to short time drift. By plotting these center frequencies as a function of time a graph called spectrogram can be produced.

For example, a $14.21 \mathrm{GHz}$ beat frequency is downconverted with a reference having a frequency of $3.3 \mathrm{MHz}$ offset of $14.21 \mathrm{GHz}$. A time trace of such a signal recorder in the oscilloscope is shown in Fig. 11a. This time traced signal is in same shape of $14.21 \mathrm{GHz}$ beat frequency. For Discrete Fourier transformation (DFT) operation, we set a time window of $20 \mu \mathrm{s}$. Time traced signal within this time frame will be taken into consideration for time domain to frequency domain conversion. A frequency spectrum of corresponding time window by DFT operation is shown in Fig. 11b, with a mean frequency at $3.3365 \mathrm{MHz}$. The center frequencies of these spectrums are plotted as a function of time, hence produced the spectrogram of this time trace, shown in Fig. 12. There is a trade-off between the width off the time window and frequency resolution of the spectrogram. Lower the time window of the DFT (as in Fig. 11a) lower the resolution of the frequency spectrum (as in Fig. 11b) but higher the frequency resolution of the spectrogram (as in Fig. 12). Obtained from various iteration duration from $1 \mathrm{~ms}$ to $100 \mathrm{~ms}$, the plot of the standard deviation is shown in Fig. 13. The plot indicates that with increase of iteration duration the locked frequency detuning shows little change and the maximum standard deviation is close to within $40 \mathrm{kHz}$.

\section{Loop response}

The experimental setup for loop response measurement is shown in Fig. 14. The response of the OFLL can be measured by disturbing the current controller (Thorlabs

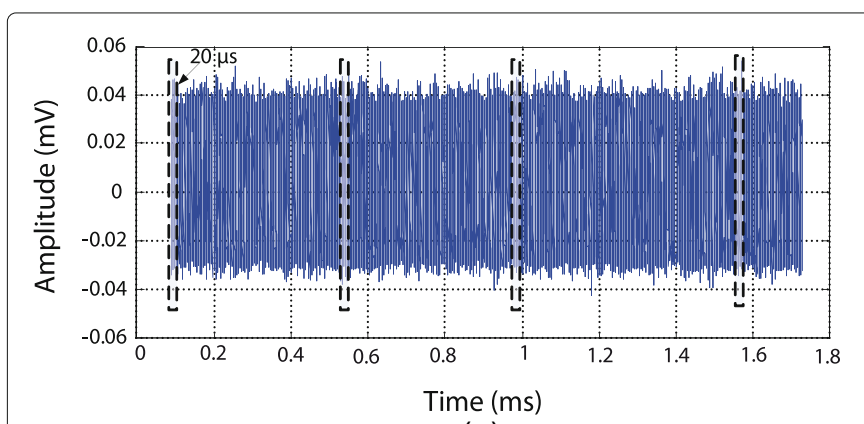

(a)

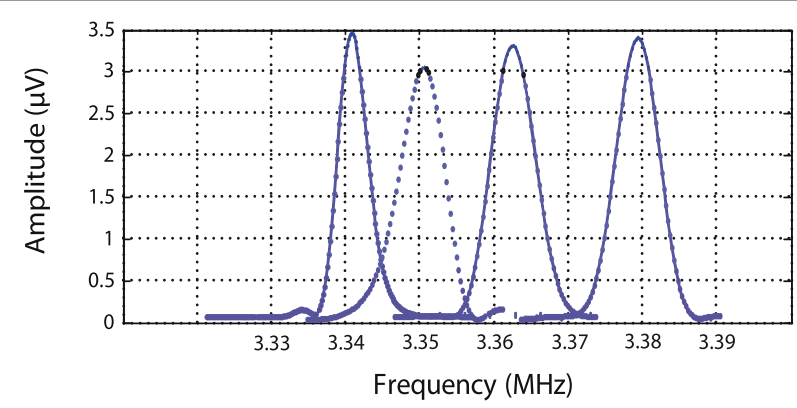

(b)

Fig. 11 Downconverted beat frequency. a time trace $(\mathbf{b})$ frequency spectrum of the time traced signal 

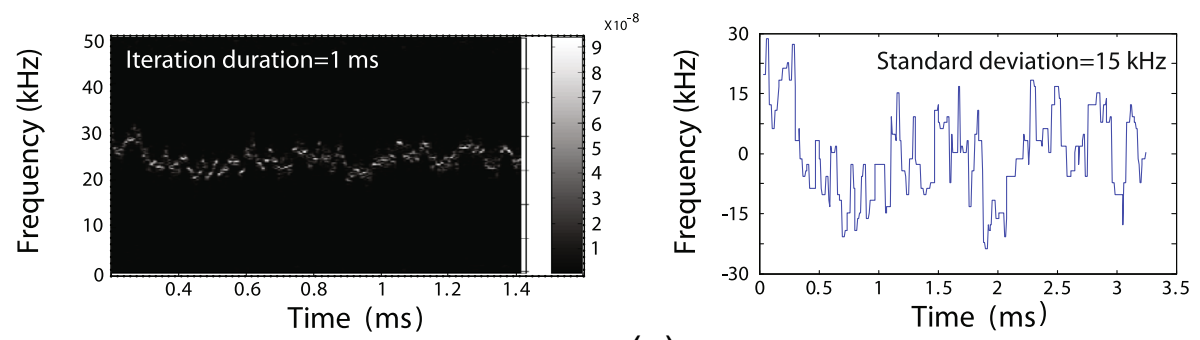

(a)
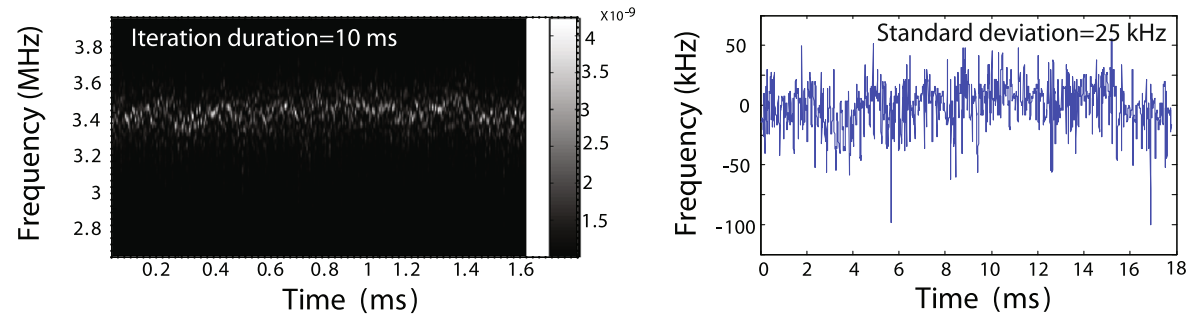

(b)
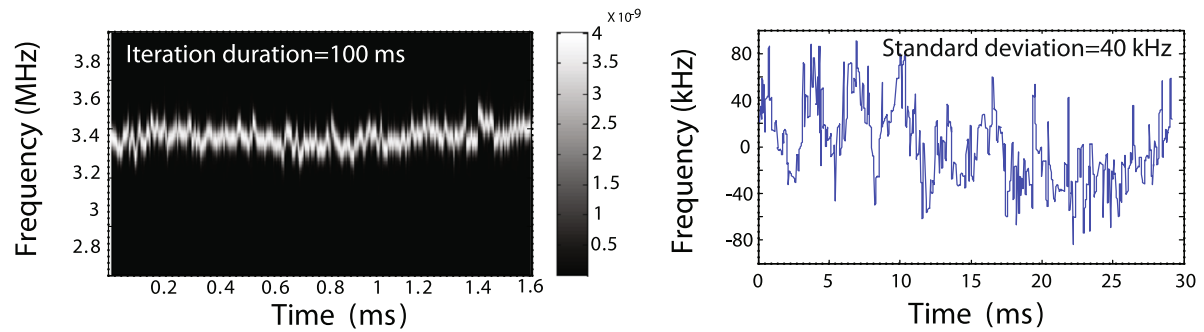

(c)

Fig. 12 Spectrogram and standard deviation around mean frequency for various short-time duration for locked condition with (a) iteration duration $1 \mathrm{~ms}$, standard deviation $15 \mathrm{kHz}(\mathbf{b})$ iteration duration $10 \mathrm{~ms}$, standard deviation $25 \mathrm{kHz}$ (c) iteration duration $100 \mathrm{~ms}$, standard deviation $40 \mathrm{kHz}$

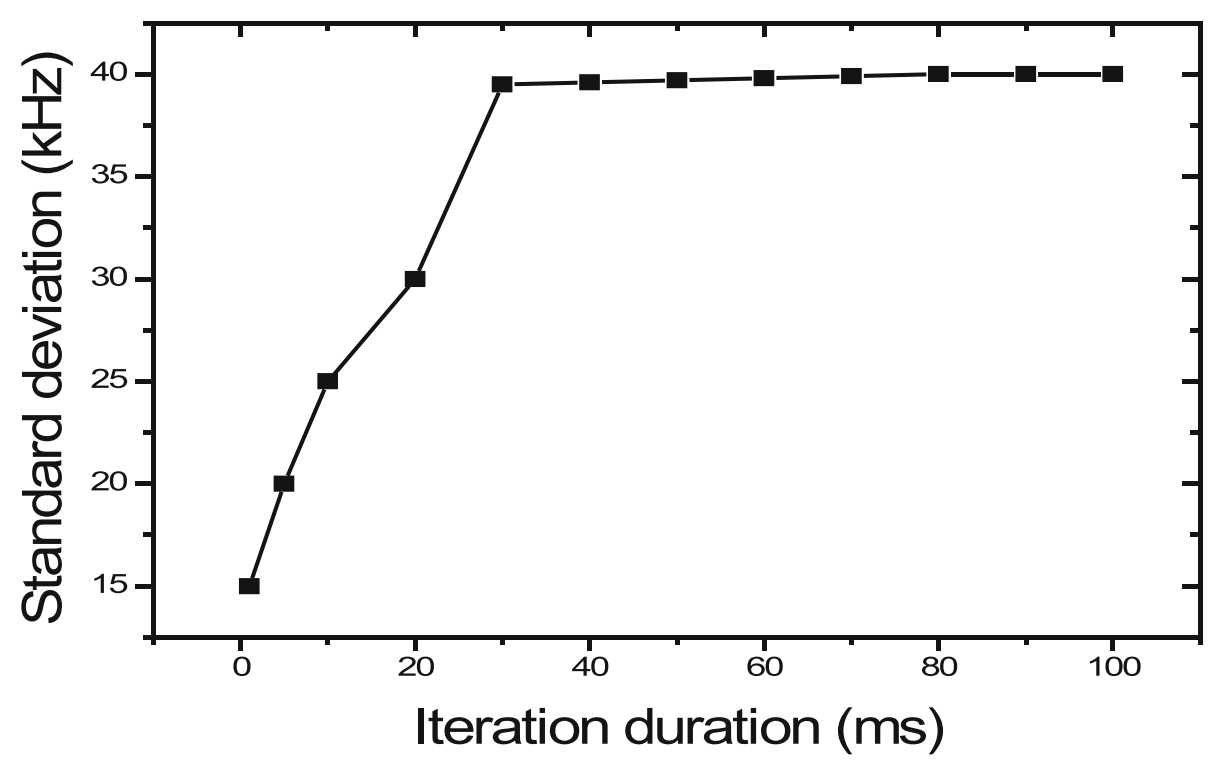

Fig. 13 Standard deviation for iteration duration from $1 \mathrm{~ms}$ to $100 \mathrm{~ms}$ 


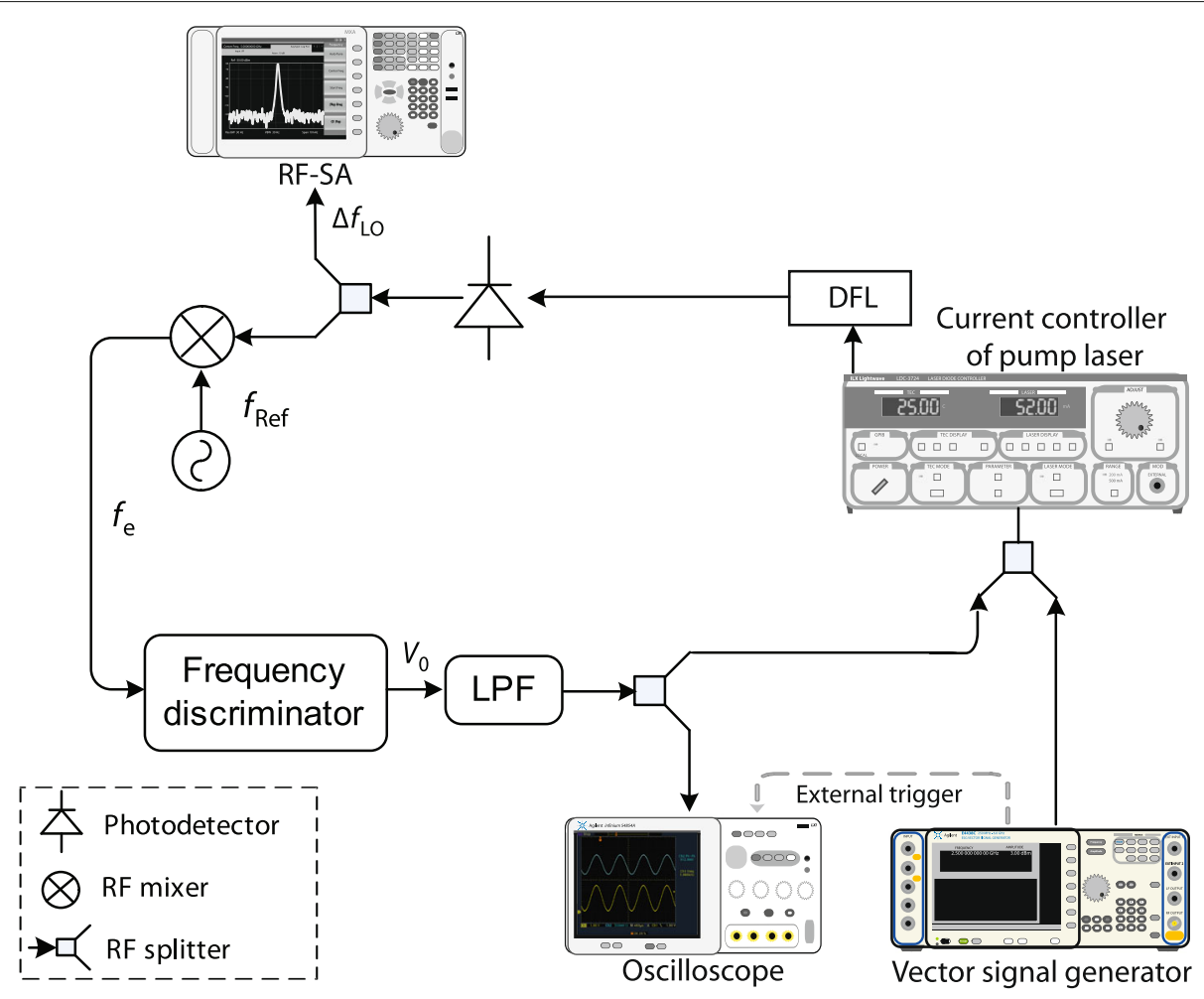

Fig. 14 Loop response time measurement setup

PRO8000) of the pump laser with a step signal from a vector signal generator (Agilent PSG E8267D) and simultaneously by measuring the resulting response at the output of the LPF. The step signal will introduce an external disturbance in the loop and the optically generated carrier signal will suffer from a temporal deviation from the desired frequency due to this temporal instability, also called ringing. Soon after the disturbance the loop will eventually bring the system in former stable condition. The recovery duration of the generated carrier signal from its unstable condition is measured by an oscilloscope (Agilent infinium 54854A). For accurate measurement the oscilloscope and the vector signal generator are synchronized by an external trigger.

The step response of the OFLL is measured for various damping factor, $\zeta$. The step responses are plotted in Fig. 15 for $\zeta=1, \zeta=0.7$ and $\zeta=0.4$.

From Eq. (11) and (12) we calculate the natural frequency $f_{\mathrm{n}}=200 \mathrm{kHz}$ and damping factor $\zeta=0.7$ for a loop gain, $K_{\mathrm{a}}$, of $3 \mathrm{~dB}$. From Eq. (11) and (12) it is noticed that an increase of gain will increase the loop natural frequency but will decrease the damping factor. These phenomena can be observed in Fig. 15. For example, with $K_{\mathrm{a}}=5 \mathrm{~dB}$ and $K_{\mathrm{a}}=1 \mathrm{~dB}$ the natural frequency and the damping factor become $f_{\mathrm{n}}=350 \mathrm{kHz}, \zeta=0.4$ and $f_{\mathrm{n}}=50 \mathrm{kHz}, \zeta=1$, respectively.
With the help of Eq. (10) we also calculated the step response of the loop using the above values of natural frequencies and damping factors. As the input of the system is an unit impulse the stabilization can be thought of as the response to a brief external disturbance. As shown in Fig. 15b, at a brief external disturbance the OFLL stabilizes in $12 \mu$ s for $\zeta=0.7$. With lower and higher damping factor the loop experiences more and less oscillation, respectively, before its stabilizes. For instance, with both $\zeta=$ 0.4 , and $\zeta=1$, the OFLL stabilizes in $14 \mu \mathrm{s}$ as shown in Fig. $15 \mathrm{a}$ and $15 \mathrm{c}$, respectively. These experimental values are in good agreement with the calculated values. From this result it is clear that the loop response time depends on the value of the damping factor, $\zeta$, and its proper choice depends on the values of gain and natural frequency.

\section{Phase noise of the generated carrier}

Besides improving the frequency stability, a degree of phase-noise reduction can be achieved by implementing the OFLL using the DFL. From Leeson's well known phase noise model for oscillator [43], random walk of frequency noise is usually very close ( $<100 \mathrm{kHz}$ offset) to the carrier [44]. It is usually related to the oscillator's physical environment (mechanical shock, vibration, temperature, or other environmental effects) [44]. It has been reported previously that the low frequency noise $(<100 \mathrm{kHz}$ offset) 


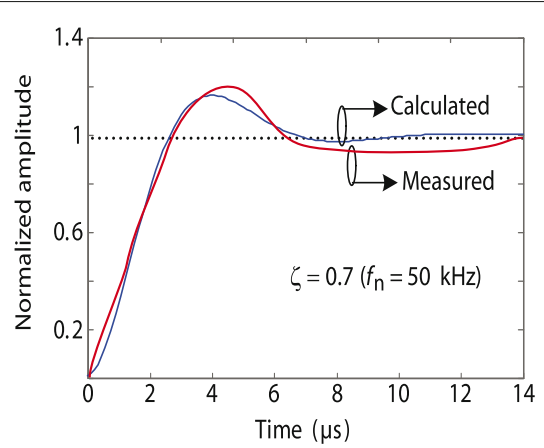

(a)

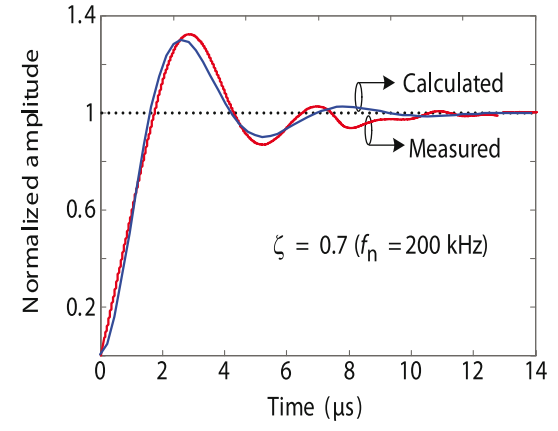

(a)

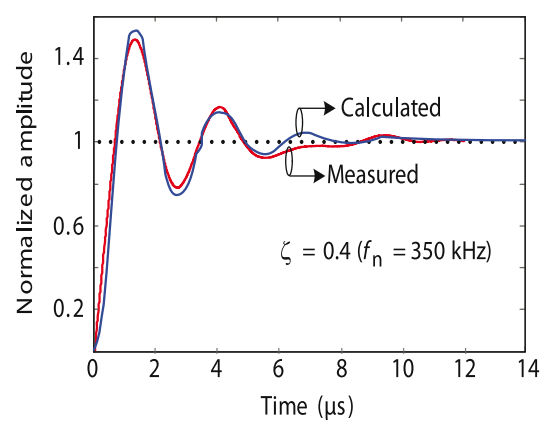

(b)

Fig. 15 Loop response time (settling time) comparison on calculated and measured values for various damping factors, $\zeta$, using step response. a $\zeta=1(\mathbf{b}) \zeta=0.7(\mathbf{c}) \zeta=0.4$

can be compensated by locking the microwave carrier generated from a laser to an ultra-low noise RF oscillator $[45,46]$. Thus an OFLL can optimize this noise effect due to environment [45].

As demonstrated in Fig. 15, the measured and calculated response of the OFLL is found to be optimized for $\zeta=0.7$ and used for frequency stabilization of the OFLL. With $\zeta=0.7$ the loop stabilizes in $12 \mu$ s. This provides a loop speed of $80 \mathrm{kHz}$. This ultimately means that the OFLL could improve the laser phase noise below an offset frequency of $80 \mathrm{kHz}$ from the carrier.

The phase noise of the generated microwave carrier has been measured for both free-running and locked condition, shown in Fig. 16. A phase noise of $-75 \mathrm{dBc} / \mathrm{Hz}$ is observed at $1 \mathrm{MHz}$ frequency offset of the $14.21 \mathrm{GHz}$ beat signal. By implementing an OFLL we obtained an improvement of the phase noise by $5 \mathrm{~dB}$ below $100 \mathrm{kHz}$ offset frequency compared to the free-running condition.

\section{Noise analysis}

The generated microwave carrier must be well above the total noise floor contributed from various noise sources, for instance thermal, shot, and relative intensity noise (RIN), to maintain a CNR specified for a particular

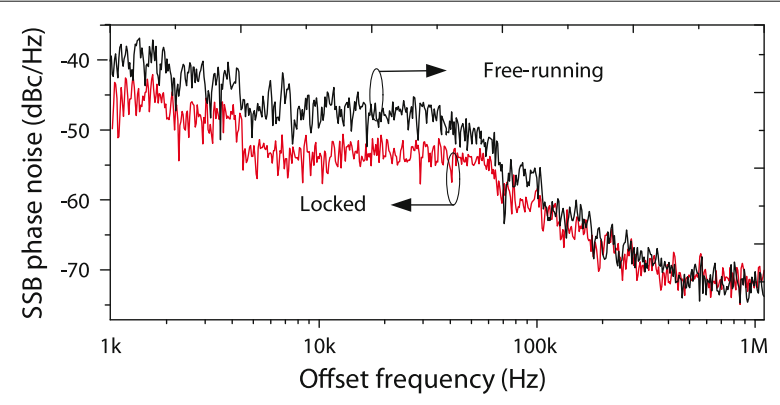

Fig. 16 Phase noise of the optically generated microwave carrier from dual frequency laser application. The system noise was characterized for various photo-current. The block diagram of the experimental setup to measure the noise floor is presented in Fig. 17.

The optical power from the pump laser was injected into the DFL and then its output was passed through the VOA before inserting into the PD. The VOA was varied from $0 \mathrm{~dB}$ to $60 \mathrm{~dB}$ with a small step and for each step the total noise power spectral density (PSD) was measured with the RF-SA using the noise marker. The marker was positioned at the frequency of $3.8 \mathrm{GHz}^{2}$ and the noise was measured in a $10 \mathrm{kHz}$ noise bandwidth. The marker gave the measured noise power normalized in $1 \mathrm{~Hz}$ bandwidth, i.e., in $\mathrm{dBm} / \mathrm{Hz}$. Due to high displayed average noise level (DANL) of the RF-SA used in our noise measurement a low-noise RF amplifier (LNA) was used to overcome the dominate noise of the RF-SA over the noise floor of the DFL. The LNA amplified the noise from the DFL above the DANL. However, the LNA itself contributed to an additional noise to the measurement. As a result, the measured noise power consists of both the amplified noise of the DFL by the LNA gain and noise from the LNA itself.

Assuming $p_{\mathrm{N} \text {,meas }}$ is the measured noise PSD in $\mathrm{W} / \mathrm{Hz}$ and $g_{\mathrm{LNA}}$ is the LNA gain on linear scale, total noise contribution from the DFL can be expressed as

$$
p_{\mathrm{N}}=\frac{p_{\mathrm{N}, \mathrm{meas}}-p_{\mathrm{LNA}}}{g_{\mathrm{LNA}}}
$$

where $p_{\mathrm{LNA}}$ is the LNA noise, related to the LNA noise factor, $F_{\mathrm{LNA}}$ having the relation

$$
p_{\mathrm{LNA}}=g_{\mathrm{LNA}} k T\left(F_{\mathrm{LNA}}-1\right)
$$

In the equation above, $k$ is the Boltzmann constant and $T=290 \mathrm{~K}$. The noise factor is related to the noise figure via the relation $\mathrm{NF}_{\mathrm{LNA}}=10 \log _{10} F_{\mathrm{LNA}}$. 


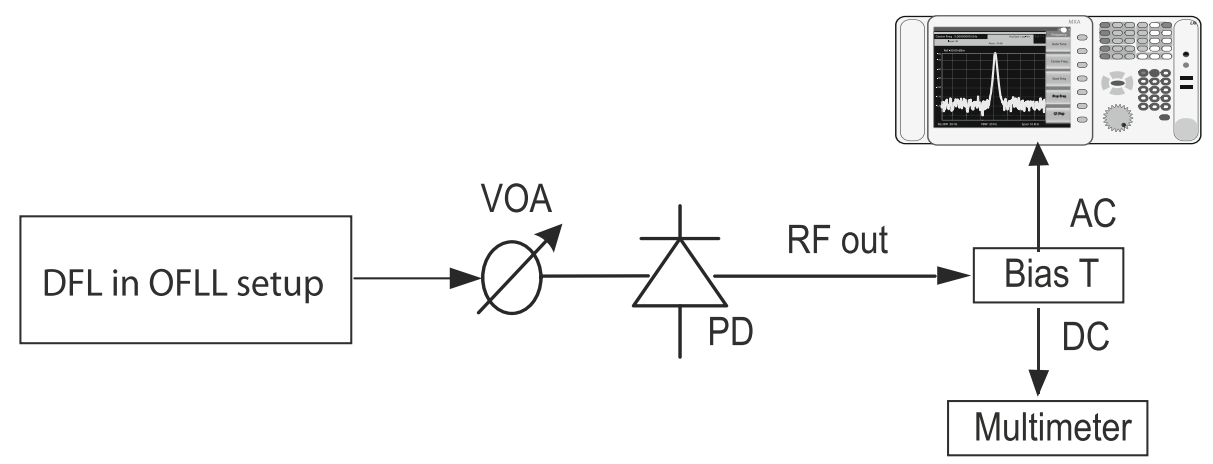

Fig. 17 Schematic of the noise floor measurement setup

Suppose that the average photocurrent during the noise measurement is $I_{\mathrm{av}}$ and the load resistance in the detector is $R_{\mathrm{L}}$, we can write the noise PSD in $\mathrm{W} / \mathrm{Hz}$, as [47]

$$
\begin{aligned}
p_{\mathrm{N}} & =p_{\text {th }}+p_{\text {shot }}+p_{\text {RIN }} \\
& =p_{\text {th }}+\frac{1}{4}\left(2 q I_{\mathrm{av}} R_{\mathrm{L}}+10^{\frac{R I N}{10}} I_{\mathrm{av}}^{2} R_{\mathrm{L}}\right)
\end{aligned}
$$

where $p_{\text {th,mL }}, p_{\text {shot }}$ and $p_{\text {RIN }}$ are the thermal noise, shot noise and RIN PSDs in $\mathrm{W} / \mathrm{Hz}$. A low noise RF amplifier (Mini-Circuits ZX60-3800LN+) with a gain of $22.5 \mathrm{~dB}$ and a noise figure of $1 \mathrm{~dB}$ at $3.8 \mathrm{GHz}$ is used during the noise measurement. The detected photocurrent was measured with a multimeter from the DC output of the bias T. Thus, substituting $p_{\mathrm{N}}$ in Eq. (16) with the expression in Eq. (14), we can determine the RIN value of the DFL. Using Eq. (16) the total noise power and individual noise contributions are plotted in Fig. 18a. The RIN value of the DFL is plotted in Fig. 18b. The measured value was extracted from the total noise PSD measurement shown in Fig. 18a. As shown, the total noise floor is dominated by the relative intensity noise (RIN) contribution.
The optical spectrum of the DFL was measured by an optical spectrum analyzer (OSA) (ANDO AQ6317) with a resolution of $0.01 \mathrm{~nm}$ and is shown in Fig. 4b. This optical spectrum was measured at a pump current of $0.8 \mathrm{~A}$. The two optical modes contain an optical power of $P_{\mathrm{L} 1}=$ $-17.5 \mathrm{dBm}(17.8 \mu W)$ and $P_{\mathrm{L} 2}=-6 \mathrm{dBm}(251 \mu W)$, respectively. The total measured optical power using an optical power meter was $P_{\mathrm{T}}=-5.7 \mathrm{dBm}(0.27 \mathrm{~mW})$. The photo-current, $I_{\mathrm{av}}$, and the RF carrier power, $P_{\mathrm{LO}}$, can be calculated by using the measured values of optical power using Eq. (5) and (6), such as $I_{\mathrm{av}}=0.11 \mathrm{~mA}, P_{\mathrm{LO}}=$ $-41 \mathrm{dBm}$ and the total optical power, $P_{\mathrm{T}}=0.27 \mathrm{~mW}$. The responsivity of the $\mathrm{PD}\left(r_{\mathrm{pd}}\right)$ as $0.4 \mathrm{~A} / \mathrm{W}$ and $R_{\mathrm{L}}=50 \Omega$ is considered.

As stated earlier, the phase noise is plotted in the decibel scale. To make better comparison between the phase noise and the noise floor of the heterodyning system, the noise floor needs to be plotted on same scaling as that of the phase noise. In order to express the noise power in decibel scale, the Fig. 18a is re-plotted with reference to the optically generated LO power (in Fig. 4a) in Fig. 18c. Hence, the PSD plot in $\mathrm{dBm} / \mathrm{Hz}$ of Fig. 18a will

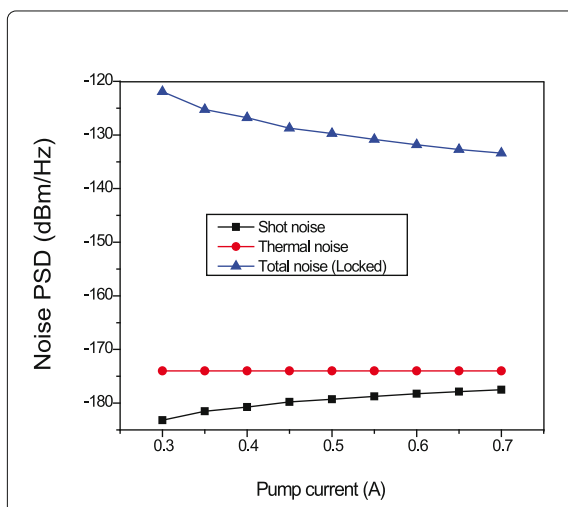

(a)

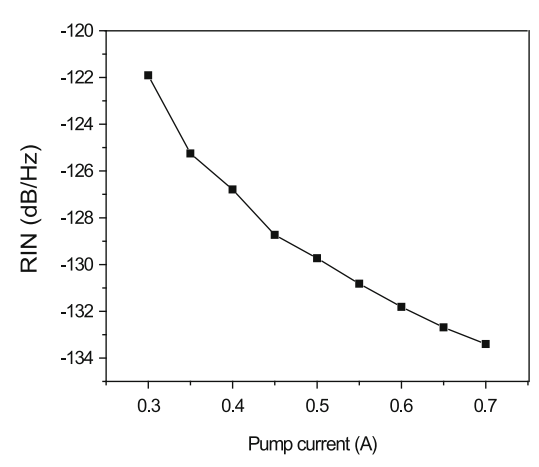

(b)

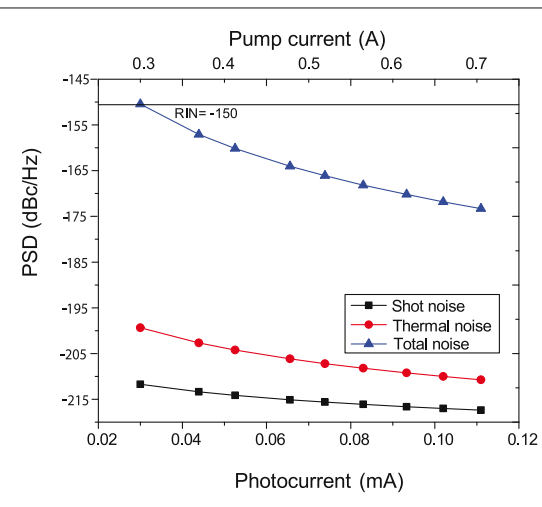

(c)

Fig. 18 Noise floor for various pump currents/photo-current. a The measured total noise PSD for the DFL consisting individual noise. b RIN measurement (c) noise power in decibel scale 
be transformed to PSD plot in $\mathrm{dBc} / \mathrm{Hz}$ as a function of the average photo-current. Given in Table I, at $1 \mathrm{MHz}$ offset frequency the generated LO should maintain a phase noise of $-110 \mathrm{dBc} / \mathrm{Hz}$, this means the noise floor of the system should be below $-110 \mathrm{dBc} / \mathrm{Hz}$. It is evident from Fig. $18 \mathrm{c}$ that at an average photo-current of $0.03 \mathrm{~mA}$ and above, the DFB laser with any RIN value will keep the total noise floor much below of $-110 \mathrm{dBc} / \mathrm{Hz}$.

With the help of Eq. (5), and taking the responsivity of the $\mathrm{PD}\left(r_{\mathrm{pd}}\right)$ as $0.4 \mathrm{~A} / \mathrm{W}$, the minimum power required for each laser is:

$$
P_{\mathrm{L}}=\frac{I_{\mathrm{av}}}{2 r_{p d}}=\frac{0.03 \times 10^{-3}}{2 \times 0.4}=0.04 \mathrm{~mW}
$$

As the optical power needs to be distributed to each PD of 25 tiles in the PAA, the minimum optical power of each laser required for 25 tiles is $1 \mathrm{~mW}(0.04 \mathrm{~mW} \times 25)$. Finally, the RF output of the PD will be distributed to 64 AE's of each tile, and should be amplified by individual RF amplifier if necessary. Having a minimum optical power of $1 \mathrm{~mW}$ from a laser with a RIN of $-122 \mathrm{~dB} / \mathrm{Hz}$, the total noise floor will be below $-110 \mathrm{dBc} / \mathrm{Hz}$. This will satisfy the minimum CNR of $110 \mathrm{~dB}$. All these parameters will ensure the optically generated $\mathrm{LO}$ which will maintain a phase noise of $-110 \mathrm{dBC} / \mathrm{Hz}$ at $1 \mathrm{MHz}$ offset frequency from the carrier frequency.

\section{Conclusions}

In the past years, reports on microwave carrier generation by using a DFL were mainly focused on very low phase noise $[48,49]$ or large tunability $[15,50]$. However, there seems to be very little attention to some crucial requirements of the generated carrier, namely its short term as well as long-term frequency stability. Our OFLL clearly addresses this crucial requirement by achieving a frequency stability of $1 \times 10^{-10}$ for averaging time of $1000 \mathrm{~s}$. At very short time iteration standard deviation for the OFLL was very low. With increase of iteration duration the locked frequency the standard deviation shows little change and close to within $40 \mathrm{kHz}$. By employing an OFLL the standard deviation of the frequency stability of the generated carrier was improved by 70 fold. Loop response time (settling time) comparison on calculated and measured values for various damping factors are also presented. These experimental values are in good agreement with the calculated values. The total noise power and individual noise contributions from the stable generated carried is also investigated. It is observed that the total noise floor is dominated by the relative intensity noise (RIN) contribution. A stable carrier of $14.21 \mathrm{GHz}$ is observed with a phase noise of $-75 \mathrm{dBc} / \mathrm{Hz}$ at $1 \mathrm{MHz}$ frequency offset. Since our DFL has great potential for integration with generic silicon-based photonic platforms, it paves the way for microwave generation on a hybrid integration platform. An integrated OFLL will be less affected by the overall environmental noise because of its reduced footprint [51], thus promising an even higher stability. The demonstrated system has a great potential to be used in application on generation and distribution of highly stable reference signal such as ALMA or space navigation tracking in the NASA Deep-space Network (DSN).

\section{Endnotes}

${ }^{1}$ This value is taken from the data sheet of commercial current controller (Thorlabs PRO8000) of the pump laser.

${ }^{2}$ The RF-SA (Agilent MXA N9020A) used in this setup gives the lowest noise floor at this frequency.

\section{Acknowledgements}

The authors gratefully acknowledge the support of the Smart Mix Programme of the Netherlands Ministry of Economic Affairs and the Netherlands Ministry of Education, Culture and Science.

Funding

Not applicable.

\section{Authors' contributions}

The original idea, mathematical framework, experiment and simulation model were developed by the first author. The second and third authors worked on the simulations and evaluation. The fourth author supervises the experimental and simulation work. The fifth author conducted the characterization of the DFL and helped to draft the manuscript. The progress is a result of common contributions and discussions. The authors also gave final approval of the version to be submitted. All authors read and approved the final manuscript.

\section{Competing interests}

The authors declare that they have no competing interests.

\section{Publisher's Note}

Springer Nature remains neutral with regard to jurisdictional claims in published maps and institutional affiliations.

Received: 8 May 2017 Accepted: 16 October 2017

Published online: 27 October 2017

\section{References}

1. Meijerink, A, Roeloffzen, CGH, Meijerink, R, Zhuang, LM, Marpaung, DAl, Bentum, MJ, Burla, M, Verpoorte, J, Jorna, P, Hulzinga, A, van Etten, W: Novel ring resonator-based integrated photonic beamformer for broadband phased array receive antennas-part I: design and performance analysis. J. Lightw. Technol. 28(1), 3-18 (2010)

2. Adler, CO, Van Alen, DJ, Carson, RS, Morrison, AF, Lavelle, ME, Anderson, ED, Onorati, GR, Cunningham, JK: Airborne reception of data and direct broadcast TV using a phased array antenna. In: Proc. IEEE Int. Conf. on Phased Array Sys. and Tec., pp. 223-226, Dana Point, (2000)

3. Marpaung, D, Zhuang, L, Burla, M, Roeloffzen, C, Verpoorte, J, Schippers, $H$ Hulzinga, A, Jorna, P, Beeker, W, Leinse, A, Heideman, R, Noharet, B, Wang, Q, Sanadgol, B, Baggen, R: Towards a broadband and squint-free $\mathrm{K}_{\mathrm{u}}$-band phased array antenna system for airborne satellite communications. In: Proc. 5th European Conf. on Antennas and Prop. (EuCAP), pp. 2623-2627, Rome, (2011)

4. Fikar, S, Scholtz, AL: Novel low-cost antenna array for vehicular reception of $\mathrm{K}_{\mathrm{u}}$-band satellite television. In: IEEE Antennas and Prop. Society Int. Symp. (AP-S), pp. 1-4, San Diego, (2008)

5. Baggen, R, Vaccaro, S, del Rio, DL: Design considerations for compact mobile $K_{\mathbf{u}}$-band satellite terminals. In: Proc. of the Second. European Conf. Antennas and Prop. (EuCAP), pp. 1-5, Edinburgh, (2007)

6. Yoshiyuki, F, Masaki, S, Seiji, N, Shinichi, T, Yutaka, O, Eiji, W, Manabu, S, Yukio, T, Tetsuya, K: Development of helicopter satellite communication 
system for the disaster information transmission. IEICE Trans. Commun. J91-B(12), 1611-1619 (2008)

7. Skolnik, M: Introduction to Radar Systems. 2nd ed. McGraw-Hill (1962)

8. VanBlaricum, ML: Photonic antenna reconfiguration: a status survey. In: Proc. of the SPIE, Photonics and Radio Freq. II. vol. 3463, pp. 180-189, San Diego

9. Ayotte, S, Babin, A, Poulin, P, Poulin, M, Jeanneau, A, Picard, MJ, Poulin, D, Davidson, CA, Aube, xM, Alexandre, I, Costin, F, Pelletier, F, Cliche, JF, Mtu, Te, Shillue, B: Laser synthesizer of the ALMA telescope: design and performance. In: Proc. IEEE Int. Topical Meeting Microwave Photonics (MWP), pp. 249-252, Montreal, (2010)

10. Payne, JM, Shillue, WP: Photonic techniques for local oscillator generation and distribution in millimeter-wave radio astronomy. In: Proc. IEEE Int. Topical Meeting Microwave Photonics (MWP), pp. 9-12, Awaji, (2002)

11. Reul, N, Tenerelli, J, Chapron, B, Vandemark, D, Quilfen, Y, Kerr, Y: SMOS satellite I-band radiometer: a new capability for ocean surface remote sensing in hurricanes. J. Geophys. Res. 117(C2), 1-24 (2012)

12. Chenakin, A: Phase noise reduction in microwave oscillators. Microwave J. 52(10), 124-140 (2009)

13. Camatel, S, Ferrero, V: Phase noise power spectral density measurement of narrow linewidth cw lasers using an optical phase-locked loop. IEEE Photon. Technol. Lett. 18(23), 2529-2531 (2006)

14. Bernhardi, EH, Khan, MRH, Roeloffzen, CGH, van Wolferen, HAGM, Wörhoff, K, de Ridder, RM, Pollnau, M: Photonic generation of stable microwave signals from a dual-wavelength $\mathrm{Al}_{2} \mathrm{O}_{3}: \mathrm{Er}^{3+}$ distributed-feedback waveguide laser. Opt. Lett. 37(2), 181-183 (2012)

15. He, $X$, Fang, $X$, Liao, C, Wang, DN, Sun, J: A tunable and switchable single-longitudinal-mode dual-wavelength fiber laser with a simple linear cavity. Opt. Exp. 17(24), 3-21781 (2009)

16. Friederich, F, Schuricht, G, Deninger, A, Lison, F, Spickermann, G, Bolivar, PH, Roskos, HG: Phase-locking of the beat signal of two distributed-feedback diode lasers to oscillators working in the $\mathrm{MHz}$ to $\mathrm{THz}$ range. Opt. Exp. 18(8), 8621-8629 (2010)

17. Tani, M, Morikawa, O, Matsuura, S, Hangyo, M: Generation of terahertz radiation by photomixing with dual- and multiple-mode lasers. Semicond. Sci. Technol. 20, S151-S163 (2005)

18. Yamada, M, Sakuda, K: Analysis of almost-periodic distributed feedback slab waveguides via a fundamental matrix approach. Appl. Opt. 26(16), 3474-3478 (1987)

19. Xia, L, Shum, P, Wang, Y, Cheng, TH: Stable triple-wavelength fiber ring laser with ultranarrow wavelength spacing using a triple-transmission-band fiber bragg grating filter. IEEE Photon. Technol. Lett. 18(20), 2162-2164 (2006)

20. Bernhardi, EH, van Wolferen, HAGM, Agazzi, L, Khan, MRH, Roeloffzen, CGH, Wörhoff, K, Pollnau, M, de Ridder, RM: Ultra-narrow-linewidth, single-frequency distributed feedback waveguide laser in $\mathrm{Al}_{2} \mathrm{O}_{3}: \mathrm{Er}^{3+}$ on silicon. Opt. Lett. 35(14), 2394-2396 (2010)

21. Huang, YC, Lin, YY: Coupled-wave theory for distributed-feedback optical parametric amplifiers and oscillators. J. Opt. Soc. Am. B. 21(21), 777-790 (2004)

22. Yang, J, Dalfsen, $\mathrm{K}$, Wörhoff, $\mathrm{K}, \mathrm{Ay}, \mathrm{F}$, Pollnau, M: High-gain $\mathrm{Al}_{2} \mathrm{O}_{3}: \mathrm{Nd}^{3+}$ channel waveguide amplifiers at $880 \mathrm{~nm}, 1060 \mathrm{~nm}$, and $1330 \mathrm{~nm}$. Appl. Phys. B. 101, 119-127 (2010)

23. Bo, L, Meng, J, Swee Chuan, T, Ping, S: Tunable microwave generation using a phase-shifted chirped fiber bragg grating. IEEE Photon. Technol. Lett. 23(18), 1292-1294 (2011)

24. Brunel, M, Amon, A, Vallet, M: Dual-polarization microchip laser at 1.53 um. Opt. Lett. 30(18), 2418-2420 (2005)

25. Khan, MRH, Burla, M, Roeloffzen, CGH, Marpaung, DAl: Phase noise analysis of an RF local oscillator signal generated by optical heterodyning of two lasers. In: Proc. of the 14th Annual Symp. of the IEEE Photonics Benelux Chapter, pp. 161-164, Brussels, (2009)

26. Lennartz, C, van Etten, W, van Osch, T, Huijskens, F: Laser spectra measured with the recirculating self heterodyne technique. J. Opt. Commun. 17(4), 138-146 (1996)

27. Ip, E, Lau, APT, Barros, DJF, Kahn, JM: Coherent detection in optical fiber systems. Opt. Exp. 16(2), 753-791 (2008)

28. Agrawal, GP: Fiber-Optic Communication Systems. Wiley, New York (1997)

29. Digital video broadcasting (DVB): Interaction channel for satellite distribution systems; guidelines for the user (EN 301 790). Technical report TR101790, V 1.1.1, European Telecommunications Standard Institute (ETSI), September 2001

30. Gardner, F: Phaselock Techniques. Wiley-Blackwell, New York (2005)

31. Yang, DH, Wang, YQ: Preliminary-results of an optically pumped cesium beam frequency standard at peking university. IEEE Trans. Instrum. Meas. 40(6), 1000-1002 (1991)

32. Casini, E, Gaudenzi, RD, Ginesi, A: DVB-S2 modem algorithms design and performance over typical satellite channels. Int. J. Satellite Commun. and Net. 22(3), 281-318 (2004)

33. Stace, T, Luiten, AN, Kovacich, RP: Laser offset-frequency locking using a frequency-to-voltage converter. Measurement Sci. Technol. 9(9), 1635-1637 (1998)

34. Castrillo, A, Fasci, E, Galzerano, G, Casa, G, Laporta, P, Gianfrani, L: Offset-frequency locking of extended-cavity diode lasers for precision spectroscopy of water at $1.38 \mu \mathrm{m}$. Opt. Exp. 18(21), 21851-21860 (2010)

35. Schunemann, $U$, Engler, H, Grimm, R, Weidemuller, M, Zielonkowski, M: Simple scheme for tunable frequency offset locking of two lasers. Rev. Sci. Instr. 70(1), 242-243 (1999)

36. Chris Toumazo, BG, Moschytz, GS (Eds): Trade-Offs in Analog Circuit Design: The Designer's Companion, 2nd ed. Kluwer (2004)

37. Stephens, DR: Phase-locked loops for wireless communications: digital, analog, and optical implementations. 2nd ed. Kluwer (2001)

38. Ryu, HY, Lee, SH, Suh, HS: Widely tunable external cavity laser diode injection locked to an optical frequency comb. IEEE Photon. Technol. Lett. 22(14), 1066-1068 (2010)

39. Rubiola, E: Phase Noise and Frequency Stability in Oscillators (Cripps, SC, ed.) Cambridge University Press (2008)

40. Sprenger, B, Zhang, J, Lu, ZH, Wang, LJ: Atmospheric transfer of optical and radio frequency clock signals. Opt. Lett. 34(7), 965-967 (2009)

41. Ning, B, Zhang, SY, Hou, D, Wu, JT, Li, ZB, Zhao, JY: High-precision distribution of highly stable optical pulse trains with $8.8 \times 10^{-19}$ instability. Sci. Rep. 4, 5109 (2014)

42. Narbonneau, F, Lours, M, Bize, S, Clairon, A, Santarelli, G, Lopez, O, Daussy, C, Amy-Klein, A, Chardonnet, C: High resolution frequency standard dissemination via optical fiber metropolitan network. Rev. Sci. Instrum 77(6), 064701-064701-8 (2006)

43. Leeson, DB: A simple model of feedback oscillator noise spectrum. Proc. IEEE. 54(2), 329-330 (1966)

44. Chorti, A, Brookes, M: A spectral model for RF oscillators with power-law phase noise. IEEE Trans. Circ. Syst. I. 53(9), 1989-1999 (2006)

45. Winter, A, Schmuser, P, Schlarb, H, Ilday, F, Kim, J-W, Chen, J, Katner, F: Phase noise characteristics of fiber lasers as potential ultra-stable master oscillators. In: Proc. of the Particle Accelerator Conference, PAC, pp. 2521-2523, Knoxville, (2005)

46. Winter, A, Schmuser, P: High-precision laser master oscillators for optical timing distribution systems in future light sources. In: Proc. of 10 th European Particle Accelerator Conference, p. 1064, Edinburgh, (2006)

47. Marpaung, D, Roeloffzen, C, Leinse, A, Hoekman, M: A photonic chip based frequency discriminator for a high performance microwave photonic link. Opt. Exp. 18(26), 27359-27370 (2010)

48. Pillet, G, Morvan, L, Brunel, M, Bretenaker, F, Dolfi, D, Vallet, M, Huignard, JP, Le Floch, A: Dual-frequency laser at $1.5 \mu \mathrm{m}$ for optical distribution and generation of high-purity microwave signals. J. Lightw. Technol. 26(13-16), 2764-2773 (2008)

49. Alouini, M, Benazet, B, Vallet, M, Brunel, M, Di Bin, P, Bretenaker, F, Le Floch, A, Thony, P: Offset phase locking of Er,Yb:glass laser eigenstates for RF photonics applications. IEEE Photon. Technol. Lett. 13(4), 367-369 (2001)

50. Gross, MC, Callahan, PT, Clark, TR, Novak, D, Waterhouse, RB, Dennis, ML: Tunable millimeter-wave frequency synthesis up to $100 \mathrm{GHz}$ by dual-wavelength brillouin fiber laser. Opt. Exp. 18(13), 13321-13330 (2010)

51. Parker, JS, Bhardwaj, A, Binetti, PRA, Hung, Y-J, Coldren, LA: Monolithically integrated gain-flattened ring mode-locked laser for comb-line generation. IEEE Photon. Technol. Lett. 24(2), 131-133 (2012) 